Research Article

\title{
Rolling Bearing Fault Diagnosis Based on Adaptive Multiparameter-Adjusting Bistable Stochastic Resonance
}

\author{
Z. H. Lai $\mathbb{D}^{1},{ }^{1}$ S. B. Wang, ${ }^{1}$ G. Q. Zhang $\mathbb{D}^{1},{ }^{1}$ C. L. Zhang, ${ }^{2}$ and J. W. Zhang ${ }^{2}$ \\ ${ }^{1}$ Guangdong Provincial Key Laboratory of Micro/Nano Optomechatronics Engineering, \\ College of Mechatronics and Control Engineering, Shenzhen University, Shenzhen 518060, China \\ ${ }^{2}$ School of Mechatronics Engineering, Nanchang University, Nanchang 330031, China \\ Correspondence should be addressed to G. Q. Zhang; zhanggq@szu.edu.cn
}

Received 18 July 2019; Revised 1 October 2019; Accepted 10 October 2019; Published 10 March 2020

Guest Editor: Franco Concli

Copyright $(2020$ Z. H. Lai et al. This is an open access article distributed under the Creative Commons Attribution License, which permits unrestricted use, distribution, and reproduction in any medium, provided the original work is properly cited.

\begin{abstract}
The weak-signal detection technologies based on stochastic resonance (SR) play important roles in the vibration-based health monitoring and fault diagnosis of rolling bearings, especially at their early-fault stage. Aiming at the parameter-fixed vibration signals in practical engineering, it is feasible to diagnose the potential rolling bearing faults through adaptively adjusting the SR system parameters, as well as other generalized parameters such as the amplitude-transformation coefficient and scale-transformation coefficient. However, extant adaptive adjustment methods focus on the system parameters, while the adjustments of other adjustable parameters have not been fully studied, thus limiting the detection performance of the adaptive SR method. In order to further enhance the detection performance of adaptive SR methods and extend their application in rolling bearing fault diagnosis, an adaptive multiparameter-adjusting SR (AMPASR) method for bistable systems based on particle swarm optimization (PSO) algorithm is proposed in this paper. This method can produce optimal SR output through adaptively adjusting multiparameters, thus realizing fault feature extraction and further fault diagnosis. Furthermore, the influence of algorithm parameters on the optimization results is discussed, and the optimization results of the Langevin system and the Duffing system are compared. Finally, we propose a weak-signal detection method based on the AMPASR of the Duffing system and employ three diagnosis examples involving inner ring fault, outer ring fault, and rolling element fault diagnoses to demonstrate its feasibility in rolling bearing fault diagnosis.
\end{abstract}

\section{Introduction}

Rolling bearings, as core components of rotating machinery, play a significant role in modern machines such as wind turbines, machine tools, centrifugal pumps, compressors, and motorized spindles. Due to their harsh and complex working environments, faults are easy to occur in the inner ring, outer ring, and rolling elements of rolling bearings, thus resulting in the breakdown of the entire machine, or even a disastrous accident. Therefore, it is necessary to monitor the rolling bearings' operating conditions and diagnose their potential faults as soon as possible, thus avoiding potential accidents through timely maintenance.
In the past few decades, various diagnosis technologies based on vibration, acoustics, temperature, and liquid have been proposed for the health monitoring and fault diagnosis of rolling bearings. Among all these diagnosis technologies, the vibration-based diagnosis technologies, such as maximum correlated kurtosis deconvolution (MCKD) [1], spectral kurtosis (SK) [2], empirical mode decomposition (EMD) [3], wavelet transform (WT) [4], and chaos theory [5], have received a lot of attention because the vibration signals with rolling bearing faults contain rich condition information. However, due to tough working environments and remote measurement distance, the fault vibration signals are always weak, especially at the fault's early stage. 
Under these conditions, the ability of weak-signal detection is a key index to evaluate the methods' diagnosis performance. Although the aforementioned diagnosis methods can achieve weak-signal detection to some extent through suppressing or canceling the noise embedded in fault vibration signals and highlighting the fault features, their weak-signal detection performances are limited because they inevitably damage the weak fault features submerged in heavy noise background in the denoising process. Compared with these noise cancellation-based fault diagnosis methods, a nonlinear phenomenon called stochastic resonance (SR) leads a type of noise utilization-based fault diagnosis methods, which have intrinsic superiority in weak-signal detection by taking advantage of the noise to enhance the weak fault features through some nonlinear systems [6].

SR was first proposed by Benzi to describe the periodicity associated with the Earth's ice ages in climatology in the 1980s [7]. This interesting nonlinear phenomenon makes it possible for the weak signals to strengthen their intensities by absorbing a certain fraction of the noise energy, thus highlighting the weak signals [8,9]. SR has attracted much attention from the physics and engineering community in the research areas such as dynamical nonlinearity [10-12], structure monitoring [13], and energy harvesting [14-16]. Moreover, in view of the good performance of SR using noise to enhance periodic signal features, SR-based weaksignal detection and fault diagnosis methods have been investigated and successfully applied in rolling bearing fault diagnosis $[17,18]$.

The performance of an SR-based weak-signal detection method is mainly determined by the form of its nonlinear systems. Research has shown that SR can appear in both monostable systems [19, 20] and bistable systems [21-23]. The latter ones, which involve the classical overdamped Langevin system and underdamped Duffing system, have much better SR performance than the former systems. Therefore, the bistable systems are mostly used as weaksignal detection models in previous studies.

SR describes an optimal synergistic action of the input signal, noise, and nonlinear systems. Therefore, aiming at the parameter-fixed noisy signals, such as the measured vibration fault signals of rolling bearings, it is possible, and the only way to adjust the system parameters, to match the signal parameters to produce SR and obtain further feature extractions. This method to achieve SR is the so-called parameter-adjusting SR method [24]. The tunable system parameters in bistable SR systems contain the potential function parameters and damping ratio (only for the underdamped systems). Furthermore, researchers found that, for the signals with inappropriate amplitude and large frequency, it is necessary to introduce amplitude-transformation coefficient and scale-transformation coefficient, respectively, to transform the amplitude and frequency of the characteristic signal to an appropriate range [25]. Combining the amplitude-transformation coefficient and scale-transformation coefficient, multiparameter-adjusting SR methods are further proposed and the multiparameters adjustment rules are fully studied theoretically [26, 27]. According to the qualitative adjustment rules, SR can be achieved by manually adjusting the multiparameters for different noisy signals.

However, the success of manual adjustments depends on the experience of engineers. The adjustment process may be time-consuming and the optimal SR output may not be obtained by engineers without sufficient experiences. This makes it an inefficient and even an unreliable way to realize SR and fault diagnosis in practical engineering. Moreover, the manual adjustment method can only be applied in offline detections. In order to solve these issues, by using some multiparameter optimization algorithms such as particle swarm optimization (PSO) [28] and genetic algorithm (GA) $[22,29]$, some adaptive parameter-adjusting methods, which can realize SR in online conditions with high efficiency and high reliability, have been proposed and studied [30-35]. These adaptive SR methods have been applied in the rolling bearing fault diagnosis successfully.

However, extant adaptive methods mainly study the adjustments of traditional system parameters, while the adjustments of other generalized parameters such as amplitude-transformation coefficient and scale-transformation coefficient have not been fully studied. Therefore, the optimal output result may not be achieved especially for those signals with a low signal-to-noise ratio (SNR), thus limiting the detection performance of the adaptive SR methods. In order to further enhance the detection performance of adaptive SR methods and extend their application in fault diagnosis, it is necessary to take the multiparameters into account and propose corresponding adaptive multiparameter-adjusting SR method. Moreover, in most references [22, 32-34, 36], the adaptive methods were adopted directly to diagnosis mechanical faults without the reliability of the optimization results being verified and the optimization parameters being discussed. Furthermore, the comparison between the adaptive fault diagnosis performances of Langevin system and Duffing system, which has not been studied yet, should be conducted that a better bistable system can be selected in further applications.

In this paper, we propose an adaptive multiparameteradjusting SR (AMPASR) method based on bistable systems. The application of this method on rolling bearing fault diagnosis is also presented. The remainder of this paper is organized as follows. In Section 2, we introduce the multiparameter-adjusting SR for both the Langevin system and the Duffing system. In Section 3, an adaptive multiparameter-adjusting SR method for bistable systems based on PSO algorithm is proposed and the adaptive optimization results are analyzed. In Section 4, we discuss the influence of algorithm parameters on the optimization results and compare the optimization results of the Langevin system and the Duffing system. Section 5 presents the procedure of the weak-signal detection based on AMPASR of the Duffing system, along with three rolling bearing fault diagnosis examples. Conclusion and summary are drawn in Section 6 . 


\section{Multiparameter-Adjusting SR for Bistable Systems}

Bistable SR systems have two typical forms. One is described by equation (1) indicating an underdamped Duffing system [31] driven by a weak signal $s(t)$ and a source of noise $n(t)$, and the other is described by equation (2) indicating an overdamped Langevin system [37] whose inertia term is ignored. In both bistable systems, $\mathrm{d} U(x) / \mathrm{d} x=-a x+b x^{3}$ denotes the force of the potential field, whose potential function $U(x)=-a x^{2} / 2+b x^{4} / 4$ with $a$ and $b$ being positive system parameters. In Duffing system equation (1) $k$ denotes the damping ratio. $s n(t)=s(t)+n(t)$ is defined as the system input signal; in the simulation analyses of this paper, $s(t)=A \cos \left(2 \pi f_{0} t\right)$ represents a harmonic weak signal with amplitude $A$ and frequency $f_{0}$, and $\sqrt{2 D} \xi(t)$ denotes a source of Gaussian white noise with noise intensity $D$ :

$$
\begin{aligned}
\ddot{x}(t)+k \dot{x}(t)-a x(t)+b x(t)^{3} & =s(t)+n(t), \\
\dot{x}(t)-a x(t)+b x(t)^{3} & =s(t)+n(t) .
\end{aligned}
$$

In the absence of both $s(t)$ and $n(t)$, the potential function in equations (1) and (2) describes a bistable potential field with two stable equilibrium points at $x= \pm \sqrt{a / b}$ and one unstable equilibrium point at $x=0$, as shown in Figure 1 . The height of the potential barrier that separates two potential wells is $\Delta U=a^{2} /(4 b)$. This is why equations (1) and (2) are called bistable systems.

The output $x(t)$ of equations (1) and (2) can be understood as the time-related position of a unit-mass Brownian particle moving in the bistable potential field $U(x)$ under the coaction of damping force $-k \dot{x}(t)$ (only for the Duffing system equation (1)), potential field force $-\mathrm{d} U(x) / \mathrm{d} x$, driving force $s(t)$ and random noise $n(t)$. Through theoretical and simulation analyses, previous research has demonstrated that SR can occur in both Duffing system equation (1) and Langevin system equation (2). That is, with the help of nonlinear systems, the noise can produce a positive effect on the weak signal $s(t)$ and enhance its intensity in the output $x(t)$. Therefore, SR in bistable systems has potential advantages in weak-signal detection.

Actually, SR presents a synergy between the weak signal, noise, and nonlinear systems. SR can only occur when these three factors are matched. For the measured vibration signal $s n(t)$ with mechanical faults, the signal parameters are fixed. Therefore, in order to utilize SR of bistable systems to detect weak fault signals, the only way is to adjust the system parameters to match the vibration signals. The SR achieved through adjusting system parameters is the so-called parameter-adjusting SR.

Traditionally, the system parameters to be adjusted involve $k, a, b$ in Duffing system equation (1) and $a, b$ in Langevin system equation (2). However, the adjustments of these system parameters are insufficient to match various noisy signals, especially for those signals with a largefrequency component, an inadequate amplitude, and a low signal-to-noise ratio (SNR). Therefore, an amplitude-

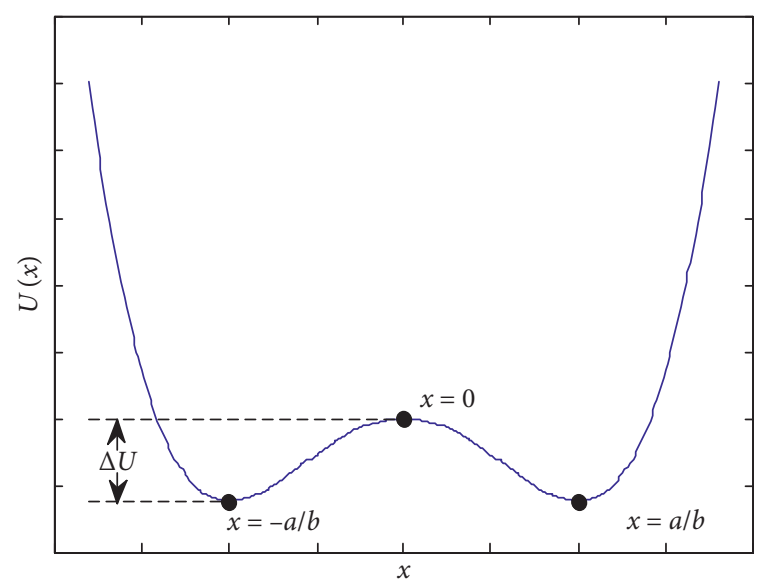

Figure 1: Bistable potential function.

transformation coefficient $\varepsilon$ and a scale-transformation coefficient $R$ are further introduced in equations (1) and (2), which can be rewritten as

$$
\begin{aligned}
\ddot{x}\left(t^{\prime}\right)+k \dot{x}\left(t^{\prime}\right)-a x\left(t^{\prime}\right)+b x\left(t^{\prime}\right)^{3} & =\varepsilon \cdot s n\left(t^{\prime}\right), \\
\dot{x}\left(t^{\prime}\right)-a x\left(t^{\prime}\right)+b x\left(t^{\prime}\right)^{3} & =\varepsilon \cdot s n\left(t^{\prime}\right) .
\end{aligned}
$$

Here, $\varepsilon$ is used to linearly transform the amplitude of the input signal to an appropriate range, and $R=\Delta t^{\prime} / \Delta t$ is used to transform the time/frequency scale of the input signal. The scale transformation is simply realized by setting the time step as $\Delta t^{\prime}=R / f_{s}$ instead of $\Delta t=1 / f_{s}$ in numerical calculations, where $f_{s}$ indicates the sampling frequency. Thus, the system output $x\left(t^{\prime}\right)$ in terms of time scale $t^{\prime}=R t$ can be obtained.

Based on Duffing system equation (3) and Langevin system equation (4), a multiparameter-adjusting method [26] has been proposed to achieve SR by jointly adjusting the generalized parameters $\mathcal{E}, R$ and system parameters $k$ (only for Duffing system), $a, b$. The SR-oriented adjustment rules for each parameter have been studied and summarized. These parameter adjustment rules have been successfully used in SR-based weak-signal detection and bearing fault diagnosis.

However, the parameter adjustment rules only show how to adjust the separate parameter to achieve SR in bistable systems. It does not reveal how to jointly adjust multiparameters, which is helpful to achieve SR for complicated and low-SNR input signals. Moreover, the existing multiparameter-adjusting method relies on the observation and manual adjustments of the engineer, and the optimal parameter sets and SR output may not be found out. Therefore, it is necessary to study an adaptive multiparameter-adjusting SR (AMPASR) method for bistable systems, which can adjust multiparameters adaptively to achieve optimal SR output for different input signals, to fully utilize the advantages of multiparameter-adjusting method for weak-signal detection and rolling bearing fault diagnosis. 


\section{AMPASR for Bistable Systems Based on PSO Algorithm}

In this section, the particle swarm optimization (PSO) algorithm will be introduced as an adaptive algorithm, and the output SNR for bistable systems will be defined as the objective function. Thus, an AMPASR method based on PSO algorithm will be proposed to achieve SR adaptively in bistable systems. The optimization results will be further analyzed in this section.

3.1. Introduction of PSO Algorithm. The PSO algorithm is an efficient multiparameter optimization algorithm. In PSO, each particle represents a potential solution to the optimization issue, and it is determined by the fitness value of the objective function. Each particle has a velocity, which determines its flight direction and distance. The velocity is dynamically adjusted by the moving experiences of the particle itself and other particles. The optimal solution will be updated according to the particles' best fitness.

The PSO algorithm can be described in mathematics. Assume that there is a population composed of $N_{p}$ particles in a $d$-dimensional solution space. The position and velocity of the $i$ th particle in the population are $X^{i}=\left(x_{i, 1}, x_{i, 2}, \ldots, x_{i, d}\right)$ and $V^{i}=\left(v_{i, 1}, v_{i, 2}, \ldots, v_{i, d}\right)$, respectively. The optimal solutions for each particle and the whole population are pbest $=P^{i}=\left(p_{i, 1}, p_{i, 2}, \ldots, p_{i, d}\right) \quad$ and $\quad$ gbest $=P_{g}=$ $\left(p_{g, 1}, p_{g, 2}, \ldots, p_{g, d}\right)$, respectively. Before the particles find out these two optimal solutions, the particle updates its velocity and position according to

$$
\begin{aligned}
v_{i, j}(q+1)= & w v_{i, j}(q)+c_{1} r_{1}\left[p_{i, j}-x_{i, j}(q)\right] \\
& +c_{2} r_{2}\left[p_{g, i}-x_{i, j}(q)\right], \\
x_{i, j}(q+1)= & x_{i, j}(q)+v_{i, j}(q+1),
\end{aligned}
$$

where $q=1,2,3, \ldots, M$ is the iteration number with $M$ the largest one; $w$ is the inertia weight; $c_{1}$ and $c_{2}$ are the positive learning factors; $r_{1}$ and $r_{2}$ are evenly distributed random numbers among $[0,1]$. In this paper, $c_{1}$ and $c_{2}$ are chosen as 2 , and $w$ is decided by an adaptive weighting method:

$$
w= \begin{cases}w_{\min }-\frac{\left(w_{\max }-w_{\min }\right) \times\left(f-f_{\min }\right)}{\left(f_{\mathrm{avg}}-f_{\min }\right)}, & f \leq f_{\mathrm{avg}}, \\ w_{\max }, & f>f_{\mathrm{avg}},\end{cases}
$$

where $w_{\max }=0.9$ and $w_{\text {min }}=0.6$ are the given maximal and minimal values of $w$; $f$ means the particle's current fitness value of the objective function, $f_{\text {avg }}$ and $f_{\text {min }}$ denote the average value and minimum value of the objective function of all particles.

With regard to the multiparameter optimization process of adaptive SR based on PSO algorithm, $d$ represents the number of multiparameters. That is, $d=5$ for the AMPASR of Duffing system (adjusting parameters include $k, a, b, \varepsilon$ and $R$ ), and $d=4$ for the AMPASR of Langevin system (adjusting parameters include $a, b, \varepsilon$ and $R$ ). As the PSO algorithm can only find out the minimal objective function among the solution space, the objective function of the optimization process is selected as the minus of output SNR, which is a commonly used SR performance index. In this paper, the input SNR and output SNR of the signals are defined as

$$
\left\{\begin{array}{l}
\mathrm{SNR}_{\mathrm{in}}=10 \log _{10}\left(\frac{\mathrm{Am}_{\mathrm{in}}^{2}}{\sum[\mathrm{SN}(f)]^{2}-\mathrm{Am}_{\text {in }}^{2}}\right), \\
\mathrm{SNR}_{\text {out }}=10 \log _{10}\left(\frac{\mathrm{Am}_{\text {out }}^{2}}{\sum[X(f)]^{2}-\mathrm{Am}_{\text {out }}^{2}}\right) .
\end{array}\right.
$$

Here, $\mathrm{SN}(f)$ represents the single-side spectrum of system input $s n(t)$, and $X(f)$ the single-side spectrum of system output $x(t)$, which can be solved by fourth-order Runge-Kutta algorithm in this paper. Thus, $\operatorname{Am}_{\text {in }}=\mathrm{SN}\left(f_{0}\right)$ and $\mathrm{Am}_{\text {out }}=X\left(f_{0}\right)$ indicate the amplitude values corresponding to characteristic signal frequency at system input and output, respectively.

Accordingly, the objective function in the multiparameter optimization of bistable SR systems is selected as $f=-\mathrm{SNR}_{\text {out }}$. Thus, the flowchart of the PSO algorithm used in this paper is presented in Figure 2.

3.2. Analysis of Adaptive Optimization Results. In this subsection, the optimal $\mathrm{SNR}_{\text {out }}$ of Duffing system equation (3) under given signal parameters will be obtained through solving the equation with different parameter sets and using the PSO algorithm, respectively. It is notable that, using the PSO algorithm, the Langevin system can produce similar simulated results, which are not presented in this paper to make it concise. Thus, the reliability and effectiveness of the PSO algorithm in AMPASR for bistable systems can be addressed.

First, the optimal $\mathrm{SNR}_{\text {out }}$ is obtained by coadjusting the system parameters $a$ and $b$. In Duffing system equation (3), the signal parameters are set as $A=0.1, f_{0}=0.01 \mathrm{~Hz}$, the sampling frequency $f_{s}=5 \mathrm{~Hz}$, and the number of sampling points $N=20000$. By setting other tunable parameters as constant $(k=0.5, \varepsilon=1$ and $R=1)$, Figures $3(\mathrm{a})$ and $4(\mathrm{a})$ present $\mathrm{SNR}_{\text {out }}$ of Duffing system against system parameters $a$ and $b$ under $D=0.4\left(\mathrm{SNR}_{\text {in }}=-22.011 \mathrm{~dB}\right)$ and $D=2.0$ $\left(\mathrm{SNR}_{\text {in }}=-29.001 \mathrm{~dB}\right)$, respectively. It can be seen from Figures 3(a) and 4(a) that the system output SNR generally presents a decreasing trend with $a$ and $b$ being increased, but the distribution rules of $\mathrm{SNR}_{\text {out }}$ values are much more complicated. The amounts of $\mathrm{SNR}_{\text {out }}$ within different ranges for Figures 3(a) and 4(a) are counted and presented in Figures 3(b) and 4(b), respectively. It can be seen that among $246 \times 246=60516 \mathrm{SNR}_{\text {out }}$ values, 426 values appear in the range of $\mathrm{SNR}_{\text {out }} \geq-8.83 \mathrm{~dB}$ in Figure 3 (a) (probability of $0.7 \%)$ and 242 values appear in the range of $\mathrm{SNR}_{\text {out }} \geq-14.7 \mathrm{~dB}$ in Figure $4(\mathrm{a})$ (probability of $0.4 \%$ ). Moreover, the largest system output SNR in Figure 3(a) appears at $a=0.24$ and $b=0.1$ with $\mathrm{SNR}_{\text {out }}=-4.8258 \mathrm{~dB}$, and the largest output SNR in Figure 4(a) appears at $a=1.52$ 


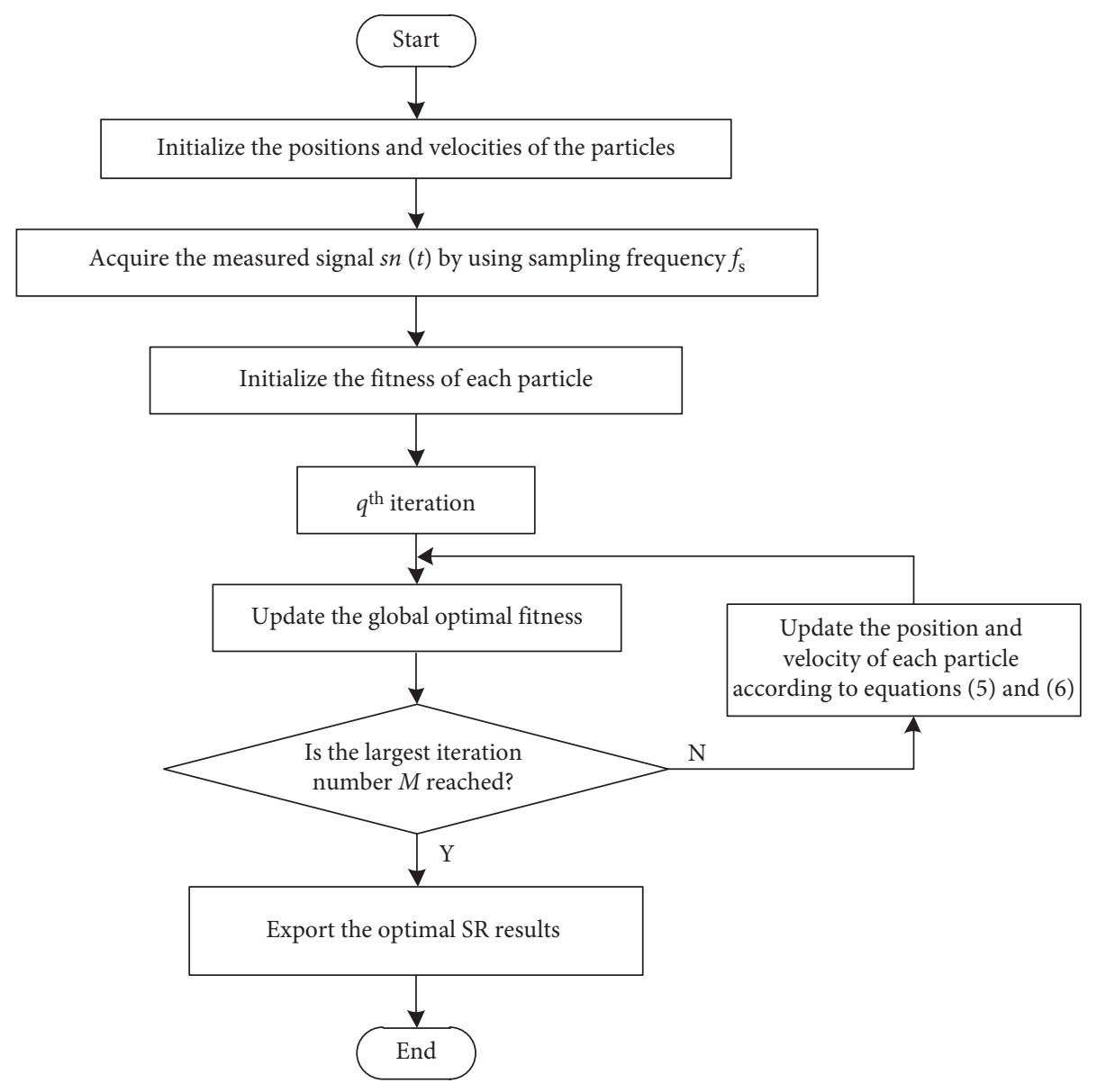

Figure 2: Flowchart of the PSO algorithm.

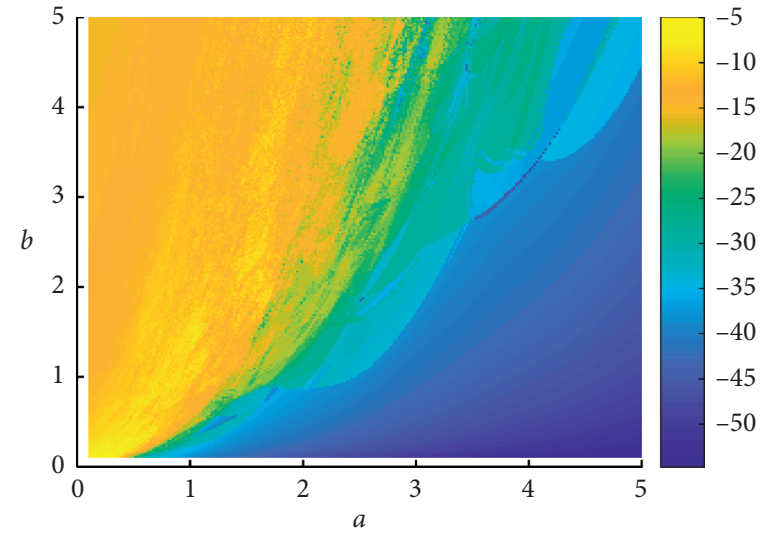

(a)

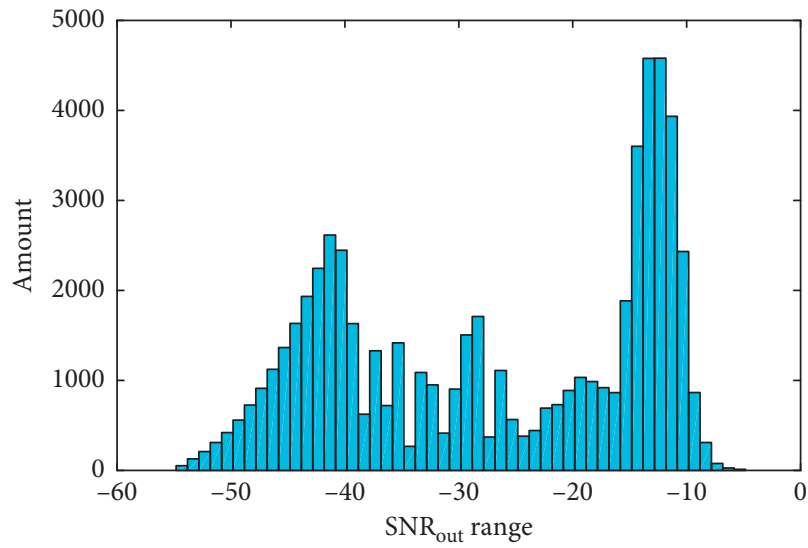

(b)

FIgURE 3: (a) $\mathrm{SNR}_{\text {out }}$ of the Duffing system against system parameters $a$ and $b$ under $D=0.4$ and (b) the distribution at different ranges.

and $b=0.56$ with $\mathrm{SNR}_{\text {out }}=-11.1684 \mathrm{~dB}$. Again, the simulation results show the significance of parameter-adjusting method in improving the output SNR of bistable systems.

Next, the optimal $\mathrm{SNR}_{\text {out }}$ is obtained through the PSO algorithm. The same signal parameters of $A=0.1$, $f_{0}=0.01 \mathrm{~Hz}$ and the sampling frequency $f_{s}=5 \mathrm{~Hz}$ are set in Duffing system equation (3). The proposed PSO algorithm, whose optimization parameters are set as $N_{p}=$ 20 and $M=20$, is used to search the optimal SNR $_{\text {out }}$ and corresponding parameters set. The searching ranges for tunable parameters are $a \in[0.1,5], b \in[0.1,5]$, while $k=0.5, \varepsilon=1$ and $R=1$ are set as those in Figures 3 and 4 . The results of 10 optimizations for $D=0.4$ and $D=2.0$ are plotted in Figure 5 with $\mathrm{SNR}_{\text {out }}$ presented in ascending order 


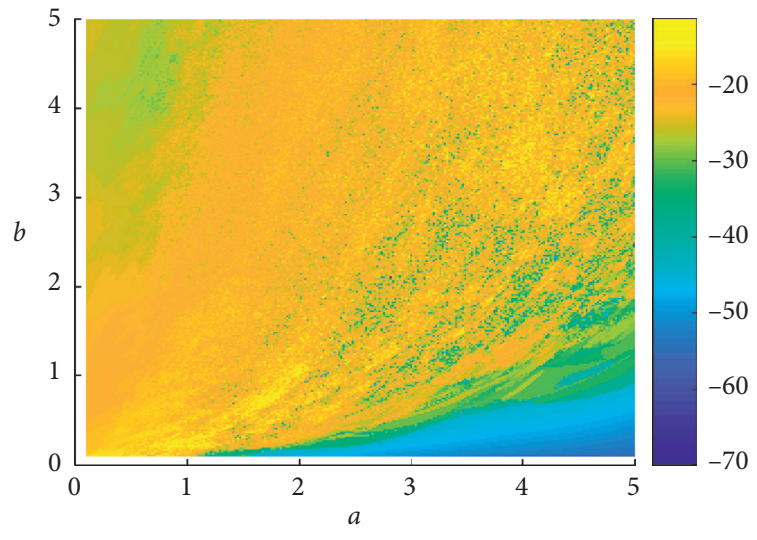

(a)

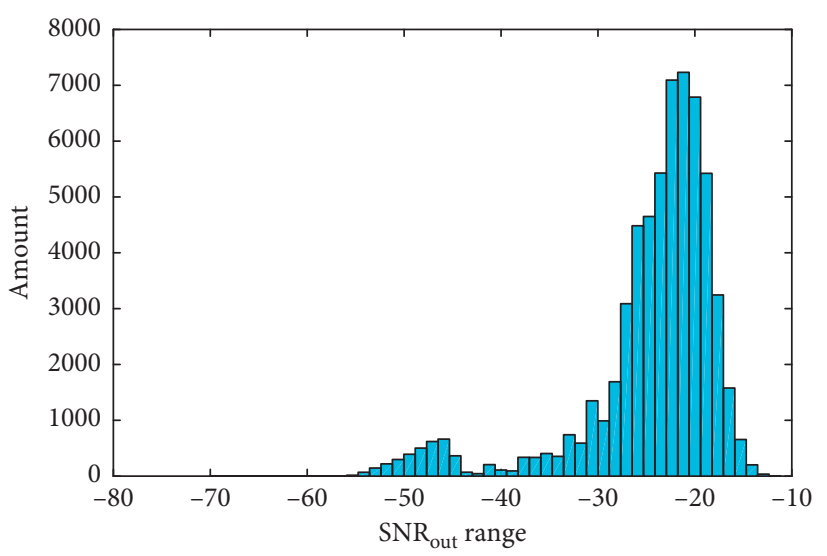

(b)

FIGURE 4: (a) $\mathrm{SNR}_{\text {out }}$ of the Duffing system against system parameters $a$ and $b$ under $D=2$ and (b) the distribution at different ranges.

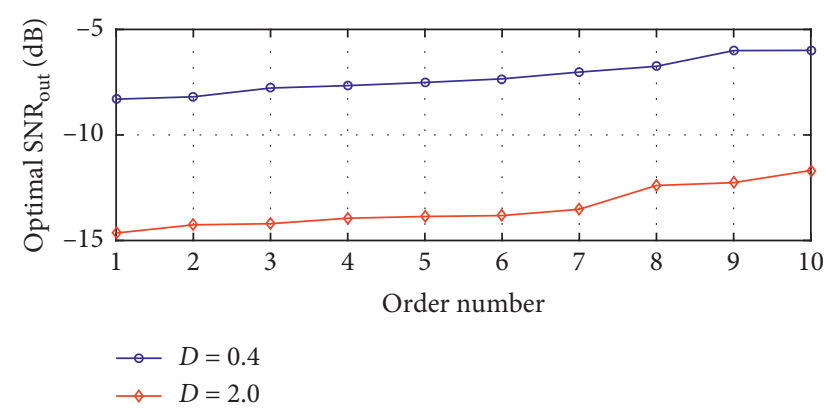

FIGURE 5: Optimal $\mathrm{SNR}_{\text {out }}$ in 10 random optimizations for $D=0.4$ and $D=2$.

and the $x$-axis indicating the order number of each optimization result.

It can be seen from Figure 5 that, in each optimization, the PSO algorithm can only find out a local optimal solution, resulting in a different optimal $\mathrm{SNR}_{\text {out }}$ in each optimization. The optimal $\mathrm{SNR}_{\text {out }}$ for $D=0.4$ in 10 random optimizations range from $-8.035 \mathrm{~dB}$ to $-5.993 \mathrm{~dB}$, which are within the $0.7 \%$ largest range of $\mathrm{SNR}_{\text {out }}\left(\mathrm{SNR}_{\text {out }} \geq-8.83 \mathrm{~dB}\right)$ compared to Figure 3(b). Similarly, the optimal $\mathrm{SNR}_{\text {out }}$ for $D=2.0$ range from $-14.65 \mathrm{~dB}$ to $-11.68 \mathrm{~dB}$, within the $0.4 \%$ largest range of $\mathrm{SNR}_{\text {out }}\left(\mathrm{SNR}_{\text {out }} \geq-14.7 \mathrm{~dB}\right)$ compared to Figure 4(a). These results indicate that although the PSO algorithm may not find the global optimal $\mathrm{SNR}_{\text {out }}$, the obtained local optimal $\mathrm{SNR}_{\text {out }}$ is quite close to the global optimal one. Thus, the proposed PSO algorithm is feasible in the optimization of AMPASR. Moreover, the relationship between $\mathrm{SNR}_{\text {out }}$ and the turntable parameters, as shown in Figures 3(a) and 4(a), is time-consuming to be obtained, while the PSO algorithm can find out an available optimal solution in a short time, thus providing a convenient method in practical applications such as online detections.

It should be pointed out that, in order to present the output results using clear three-dimensional images such as Figures 3(a) and 4(a), only two adjusting parameters ( $a$ and b) are considered in this subsection. Similar results are also obtained and show that PSO algorithm can find out an available optimal solution in a short time for all adjusting parameters.

\section{Discussion}

4.1. Influence of PSO Algorithm Parameters on the Optimization Results. In the PSO algorithm stated in Section 3.1, the particles' number $N_{p}$ and largest iteration number $M$ are preset. In this subsection, through analyzing the optimal $\mathrm{SNR}_{\text {out }}$ of a Duffing system, the influences of these two parameters on the output SR performance of bistable systems are discussed.

The selection of the adjusting parameters' searching range should be stated first. The search range of $k$ in Duffing system depends on the noise density of the input signal. The searching ranges of $\varepsilon$ and $R$ are determined by guaranteeing the amplitude and frequency of the characteristic signal component after transformation within the appropriate small-parameter range, while the searching ranges of systems $a$ and $b$ can be as large as possible. Without loss of generality, the upper limits of $a$ and $b$ are set as 5 in this section for the comparison of the optimization results.

Again, in Duffing system equation (3), the signal parameters are set as $A=0.1, f_{0}=0.01 \mathrm{~Hz}, D=2$ $\left(\mathrm{SNR}_{\mathrm{in}}=-29.001 \mathrm{~dB}\right)$, and the sampling frequency $f_{s}=5 \mathrm{~Hz}$, sampling points $N=20000$. The PSO algorithm is adopted to search the $\mathrm{SNR}_{\text {out }}$ in the searching ranges of $k \in[0.1,5], \quad a \in[0.1,5], \quad b \in[0.1,5], \quad \varepsilon \in[0.8,1.2]$ and $R \in[0.5,2]$. It is necessary to first state that the computer used for numerical simulations has CPU of Intel(R) Core(TM) i7-6700 and RAM with $8.00 \mathrm{~GB}$ as its main configurations.

First, the influence of $N_{p}$ on the system optimization results is studied. Table 1 presents the optimal $\mathrm{SNR}_{\text {out }}$ and iteration time with $M=20$ and different $N_{p}$ in 8 random optimizations. The optimization results are also plotted in Figure 6 with the data being in ascending order. It can be seen from Table 1 and Figure 6(a) that, in 8 random optimizations with different $N_{p}$, the obtained optimal $\mathrm{SNR}_{\text {out }}$ are within a narrow range around $-9.5 \mathrm{~dB}$, which is 
TAble 1: Optimization results with $M=20$ and different $N_{p}$.

\begin{tabular}{|c|c|c|c|c|c|c|c|c|c|}
\hline \multicolumn{2}{|c|}{ Number of optimization } & 1 & 2 & 3 & 4 & 5 & 6 & 7 & 8 \\
\hline$N_{p}=10$ & $\begin{array}{l}\text { Optimal } \mathrm{SNR}_{\text {out }}(\mathrm{dB}) \\
\text { Iteration time }(\mathrm{s})\end{array}$ & $\begin{array}{l}-9.188 \\
13.304\end{array}$ & $\begin{array}{l}-9.562 \\
13.272\end{array}$ & $\begin{array}{l}-9.649 \\
13.071\end{array}$ & $\begin{array}{l}-9.636 \\
13.476\end{array}$ & $\begin{array}{l}-9.478 \\
13.031\end{array}$ & $\begin{array}{l}-9.747 \\
13.336\end{array}$ & $\begin{array}{l}-9.111 \\
13.187\end{array}$ & $\begin{array}{l}-9.389 \\
13.257\end{array}$ \\
\hline$N_{p}=20$ & $\begin{array}{l}\text { Optimal } \mathrm{SNR}_{\text {out }}(\mathrm{dB}) \\
\text { Iteration time }(\mathrm{s})\end{array}$ & $\begin{array}{l}-9.513 \\
25.717\end{array}$ & $\begin{array}{l}-9.069 \\
25.974\end{array}$ & $\begin{array}{l}-9.645 \\
25.214\end{array}$ & $\begin{array}{l}-9.523 \\
25.891\end{array}$ & $\begin{array}{l}-9.534 \\
25.865\end{array}$ & $\begin{array}{l}-9.637 \\
26.278\end{array}$ & $\begin{array}{l}-9.587 \\
26.075\end{array}$ & $\begin{array}{l}-9.552 \\
26.232\end{array}$ \\
\hline$N_{p}=30$ & $\begin{array}{l}\text { Optimal SNR } \mathrm{Sut}_{\text {out }}(\mathrm{dB}) \\
\text { Iteration time }(\mathrm{s})\end{array}$ & $\begin{array}{l}-9.127 \\
38.780\end{array}$ & $\begin{array}{l}-9.147 \\
38.872\end{array}$ & $\begin{array}{l}-9.534 \\
38.581\end{array}$ & $\begin{array}{l}-9.530 \\
38.846\end{array}$ & $\begin{array}{l}-9.672 \\
38.810\end{array}$ & $\begin{array}{l}-9.269 \\
38.820\end{array}$ & $\begin{array}{l}-9.197 \\
39.054\end{array}$ & $\begin{array}{l}-9.531 \\
38.800\end{array}$ \\
\hline
\end{tabular}

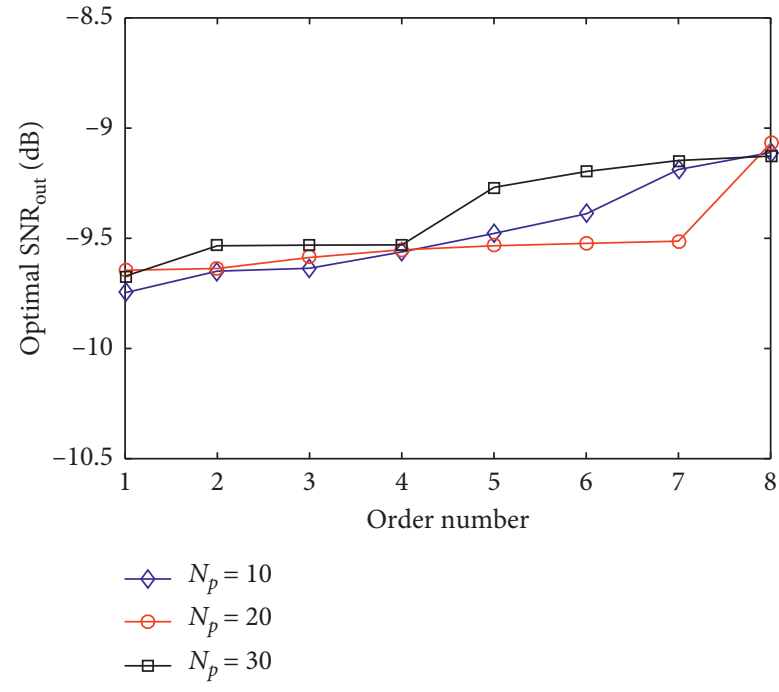

(a)

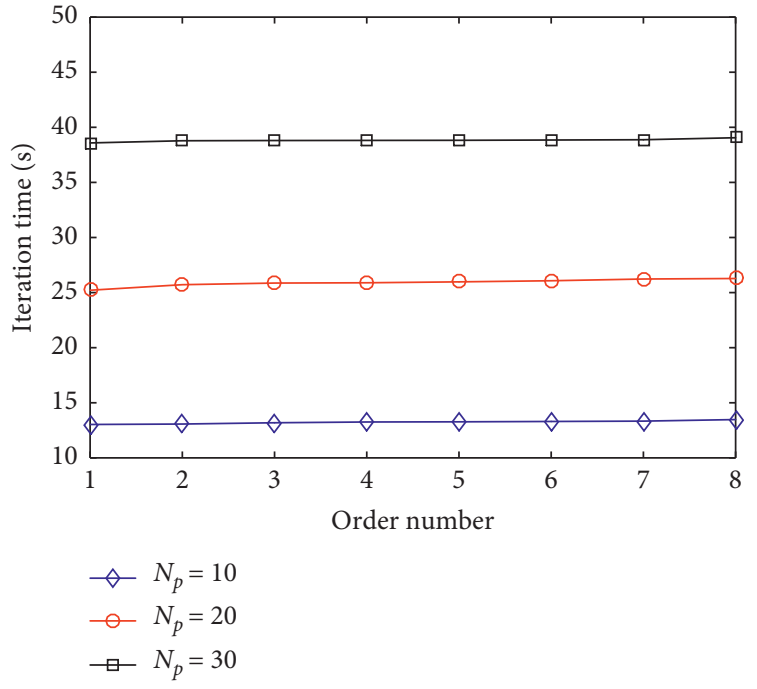

(b)

Figure 6: (a) Optimal $\mathrm{SNR}_{\text {out }}$ and (b) iteration time in 8 random optimizations with different $N_{p}$.

significantly enhanced compared to $\mathrm{SNR}_{\mathrm{in}}$, indicating that the value of $N_{p}$ barely affects the optimization results. However, Table 1 and Figure 6(b) show that, with the value of $N_{p}$ increasing, the iteration time significantly increases, thus reducing the optimization efficiency. Therefore, a small value of $N_{p}$, such as 10 , is chosen in this paper in PSO optimizations with the acceptable optimization results and short iteration time being guaranteed.

Similarly, the influence of $M$ on the system optimization results is studied as well. Table 2 and Figure 7 present the optimal SNR $_{\text {out }}$ and iteration time with $N_{p}=10$ and different $M$ in 8 random optimizations. It can be seen from Figure 7(a) that the optimal $\mathrm{SNR}_{\text {out }}$ with $M=20$ and $M=$ 30 are close and obviously better than those with $M=10$, indicating that the largest iteration number $M$ affects the optimization results when it is small, while when it is large enough, its influence can be ignored. Therefore, in this paper $M=20$ is chosen as an appropriate value of the largest iteration number that can produce acceptable optimization results in an appropriate iteration time.

4.2. Comparison between Optimization Results of Langevin System and Duffing System. Duffing system (3) and Langevin system (4) are two common used bistable systems. Previous research shows that Duffing system has better adaptability to signals with large noise intensity due to the tunable damping ratio [25]. In this subsection, PSO algorithm is used for both systems to obtain their optimal output SNR under three groups of the same signals, which are given by $A=0.1$, $f_{0}=0.01 \mathrm{~Hz}, \quad D=0.4,2,5$, the sampling frequency $f_{s}=5 \mathrm{~Hz}$, and the number of sampling points $N=20000$ in equations (3) and (4). Thus, comparison of the optimization results is further presented.

In the optimization process of PSO algorithm, $N_{p}=10$ and $M=20$ are selected based on the analysis in Section 4.1, and the search ranges for equations (3) and (4) are $k \in[0.1,5]$ (only for Duffing system (3)), $a \in[0.1,5]$, $b \in[0.1,5], \varepsilon \in[0.8,1.2]$ and $R \in[0.5,2]$, respectively. The optimization results of Duffing system and Langevin system are recorded in Tables 3 and 4, respectively. It can be seen from Tables 3 and 4 that when the noise intensity is small $\left(D=0.4, \mathrm{SNR}_{\text {in }}=-22.011 \mathrm{~dB}\right)$, the optimal $\mathrm{SNR}_{\text {out }}$ and average $\mathrm{SNR}_{\text {out }}$ in both systems are in the same level, indicating that the Duffing system and Langevin system provide similar optimization results under small noise intensity conditions. However, when the noise intensity increases $\left(D=2.0, \quad \mathrm{SNR}_{\mathrm{in}}=-29.001 \mathrm{~dB}\right.$ and $D=5.0$, $\mathrm{SNR}_{\text {in }}=-32.980 \mathrm{~dB}$ ), the optimal $\mathrm{SNR}_{\text {out }}$ of Duffing system is larger than that of Langevin system, and more importantly, the average $\mathrm{SNR}_{\text {out }}$ of Duffing system is much larger than that of Langevin system, indicating that the Duffing system can produce a desirable $\mathrm{SNR}_{\text {out }}$ more easily by using $\mathrm{PSO}$ algorithm. These can explain that the damping ratio $k$ in 
TABLE 2: Optimization results with $N_{p}=10$ and different $M$.

\begin{tabular}{|c|c|c|c|c|c|c|c|c|c|}
\hline \multicolumn{2}{|c|}{ Number of optimization } & 1 & 2 & 3 & 4 & 5 & 6 & 7 & 8 \\
\hline$M=10$ & $\begin{array}{l}\text { Optimal SNR } \mathrm{Sut}_{\text {out }}(\mathrm{dB}) \\
\text { Iteration time }(\mathrm{s})\end{array}$ & $\begin{array}{c}-9.047 \\
7.085\end{array}$ & $\begin{array}{c}-9.822 \\
6.987\end{array}$ & $\begin{array}{c}-9.563 \\
6.886\end{array}$ & $\begin{array}{c}-9.512 \\
7.069\end{array}$ & $\begin{array}{c}-9.964 \\
7.188\end{array}$ & $\begin{array}{c}-9.836 \\
6.995\end{array}$ & $\begin{array}{c}-9.539 \\
7.195\end{array}$ & $\begin{array}{c}-9.831 \\
6.938\end{array}$ \\
\hline$M=20$ & $\begin{array}{c}\text { Optimal } \mathrm{SNR}_{\text {out }}(\mathrm{dB}) \\
\text { Iteration time }(\mathrm{s})\end{array}$ & $\begin{array}{l}-9.188 \\
13.304 \\
\end{array}$ & $\begin{array}{l}-9.562 \\
13.272 \\
\end{array}$ & $\begin{array}{l}-9.649 \\
13.071 \\
\end{array}$ & $\begin{array}{l}-9.636 \\
13.476 \\
\end{array}$ & $\begin{array}{l}-9.478 \\
13.031 \\
\end{array}$ & $\begin{array}{l}-9.747 \\
13.336 \\
\end{array}$ & $\begin{array}{l}-9.111 \\
13.187 \\
\end{array}$ & $\begin{array}{l}-9.389 \\
13.257 \\
\end{array}$ \\
\hline$M=30$ & $\begin{array}{c}\text { Optimal SNR } \\
\text { out }(\mathrm{dB}) \\
\text { Iteration time }(\mathrm{s})\end{array}$ & $\begin{array}{l}-9.747 \\
19.320\end{array}$ & $\begin{array}{l}-9.422 \\
19.315\end{array}$ & $\begin{array}{l}-9.672 \\
19.056\end{array}$ & $\begin{array}{l}-9.404 \\
19.168\end{array}$ & $\begin{array}{l}-9.216 \\
19.189\end{array}$ & $\begin{array}{l}-9.573 \\
18.953\end{array}$ & $\begin{array}{l}-9.637 \\
19.148\end{array}$ & $\begin{array}{l}-9.251 \\
18.837\end{array}$ \\
\hline
\end{tabular}

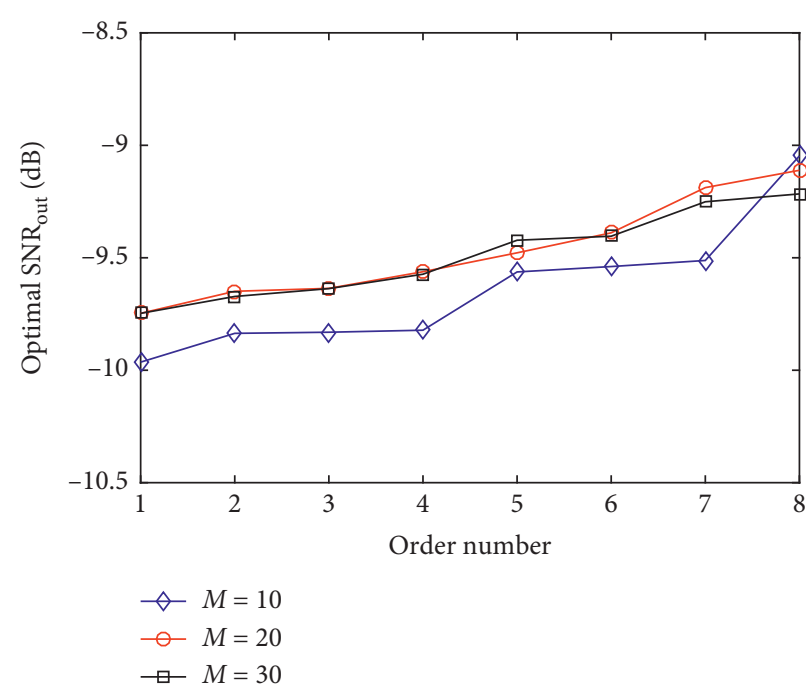

(a)

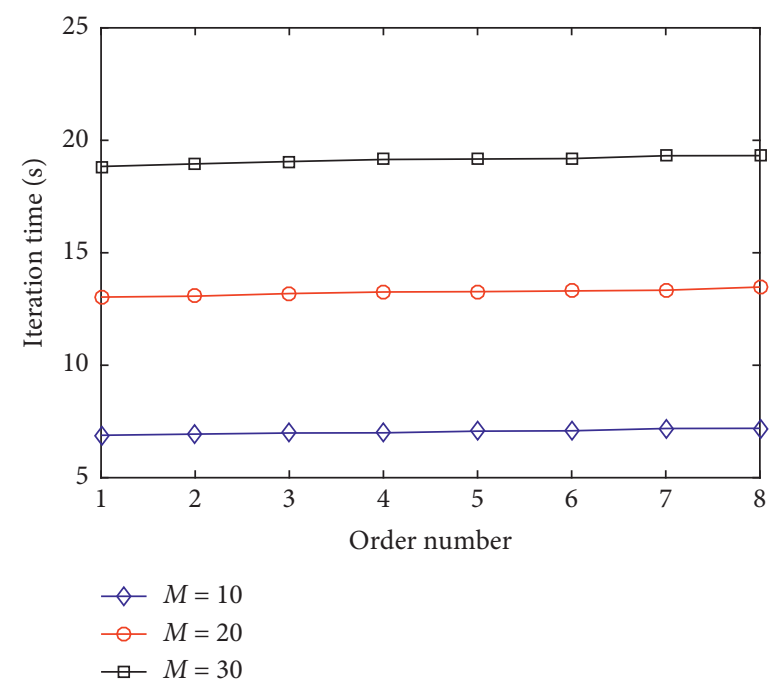

(b)

Figure 7: (a) Optimal $\mathrm{SNR}_{\text {out }}$ and (b) iteration time in 8 random optimizations with different $M$.

TABLE 3: Optimal SNR ${ }_{\text {out }}$ with detailed multiparameters and average $\mathrm{SNR}_{\text {out }}$ in 10 random optimizations of underdamped Duffing system.

\begin{tabular}{cccccccc}
\hline & $k$ & $a$ & $b$ & $\varepsilon$ & $R$ & Optimal SNR $_{\text {out }}(\mathrm{dB})$ & Average SNR $_{\text {out }}(\mathrm{dB})$ \\
\hline$D=0.4$ & 3.3375 & 0.7143 & 3.5740 & 1.0434 & 1.4005 & -2.6143 & -2.6765 \\
$D=2.0$ & 3.4443 & 0.9636 & 1.5235 & 1.1546 & 0.7427 & -9.1382 & -9.5464 \\
$D=5.0$ & 2.6534 & 2.7778 & 4.4662 & 1.0798 & 0.6425 & -11.5614 & -12.2489 \\
\hline
\end{tabular}

TABLE 4: Optimal $\mathrm{SNR}_{\text {out }}$ with detailed multiparameters and average $\mathrm{SNR}_{\text {out }}$ in 10 random optimizations of overdamped Langevin system.

\begin{tabular}{ccccccc}
\hline & $a$ & $b$ & $\varepsilon$ & $R$ & Optimal SNR $_{\text {out }}(\mathrm{dB})$ & Average SNR \\
\hline$D=0.4$ & 0.5333 & 2.1228 & 0.8955 & 0.5496 & -2.5486 & -2.7745 \\
$D=2.0$ & 1.2747 & 2.0325 & 0.8182 & 0.5148 & -9.8826 & -10.6045 \\
$D=5.0$ & 1.4025 & 0.6228 & 0.8599 & 0.5805 & -11.7765 & -14.4230 \\
\hline
\end{tabular}

the Duffing system is helpful to deal with the large noise intensity signals. Moreover, the simulation processes show that divergence may easily occur in the optimization of Langevin system, and an optimal $\mathrm{SNR}_{\text {out }}$ may not be obtained in some cases, which is another shortage of Langevin system compared to Duffing system.

Based on the previous analyses, the Duffing system, which will be selected in this paper for adaptive fault diagnosis, is suggested as a better bistable system in weaksignal detection.

\section{Practical Examples}

5.1. Weak-Signal Detection Method Based on AMPASR of the Duffing System. According to previous analyses, the AMPASR of Duffing system provides a solution for the adaptive detection of weak signals emerged in a strong noise background. One potential application of the proposed method is the diagnosis of rolling bearing incipient faults, whose vibration signals $s n(t)$ are generally noisy signals with low SNR. Considering that a steady-state $s n(t)$ in practical 
engineering can be decomposed into some periodic signals and a source of noise, the AMPASR of Duffing system proposed in this paper can be used to produce optimal SR for $s n(t)$ adaptively. Thus, the existence of the incipient fault can be judged.

In practical vibration fault signals, the accurate characteristic frequency $f_{0}$ of the faults is always unknown. Therefore, the definition of the output SNR should be modified by estimating the potential frequency domain $\left[f_{l}, f_{r}\right]$ of the fault signal. The modified $\mathrm{SNR}_{\text {out }}$, which is chosen as the objective function in the adaptive detection in practical applications, is given by

$$
\mathrm{SNR}_{\text {out }}=10 \log _{10}\left(\frac{\max \left\{\left.X(f)\right|_{f_{l}<f<f_{r}}\right\}^{2}}{\sum[X(f)]^{2}-\max \left\{\left.X(f)\right|_{f_{l}<f<f_{r}}\right\}^{2}}\right) .
$$

Thus, the procedure of bearing fault diagnosis by using the AMPASR of Duffing system, which corresponds to equation (3), is presented in Figure 8. In the procedure, the fault frequency domain $\left[f_{l}, f_{r}\right]$ should be estimated first, then an appropriate sampling frequency $f_{s}$ should be chosen to acquire the vibration signal $s n(t)$. Before optimizations, the search range of multiparameters should be determined according to the vibration signal. In what is following in this section, three fault diagnosis examples of rolling bearings are presented to demonstrate the effectiveness and reliability of the proposed method.

5.2. Adaptive Diagnosis Examples of Rolling Bearing Faults. The rolling bearing fault signals proposed in this subsection are cited from the Case Western Reserve University (CWRU) Bearing Data Center, which provides data presenting various degrees of difficulty for diagnosis [38]. The basic layout of the test rig is shown in Figure 9. It consists of a 2-horsepower reliance electric motor driving a shaft on which a torque transducer and encoder are mounted. Torque is applied to the shaft via a dynamometer and electronic control system.

In the tests, motor bearings were seeded with faults using electrodischarge machining (EDM). Faults ranging from 0.007 inches in diameter to 0.040 inches in diameter were introduced separately at the inner ring, outer ring, and rolling element. Faulted bearing was reinstalled into the test motor and vibration data was recorded for motor loads of 0 to 3 horsepower, and acceleration data was measured at locations near to and remote from the motor bearings.

In this subsection, the fault vibration signals from the drive end bearing with a motor load of 1 horsepower are used for verification. The rolling bearing is a deep groove ball bearing with the type of SKF 6205-2RS JEM. The geometric parameters and fault frequencies of the bearing are provided in 5. For the vibration signals with faults, characteristic frequencies $f_{\mathrm{BPFI}}, f_{\mathrm{BPFO}}$, and $f_{\mathrm{BPFB}}$ are expected in the signal spectrums when the fault appears in inner ring, outer ring, and rolling element, respectively. During the test, an accelerometer was placed at the 12-o'clock position at the drive end of the motor housing; the approximate motor speed was $1774 \mathrm{rpm}(29.57 \mathrm{~Hz})$ and vibration signals were collected using a 16-channel DAT recorder at 48,000 samples per second $\left(f_{s}=48000 \mathrm{~Hz}\right)$ for the drive end faults, and the data length is $N=10000$.

5.2.1. Case 1: Inner Ring Fault Diagnosis. The fault frequency of an inner ring fault can be calculated as $f_{\mathrm{BPFI}} \approx 160.1 \mathrm{~Hz}$ according to Table 5. The waveform and spectrum of the original vibration signal with inner ring fault, which is 0.021 inches in diameter indicating a severe fault condition, are presented in Figures 10(a) and 10(b). It can be seen that, due to the low input SNR $\left(\mathrm{SNR}_{\text {in }}=-34.99 \mathrm{~dB}\right)$, the signal component of inner ring fault frequency $f_{\mathrm{BPFI}} \approx 160.1 \mathrm{~Hz}$ is submerged in the waveform and is not evident compared to other signal components in the spectrum. Thus, the inner ring fault signal hidden in the original vibration signal cannot be identified.

In order to detect the potential inner ring fault of the rolling bearing, the proposed AMPASR method of Duffing system is adopted to analyze the vibration signal. In each optimization, the potential frequency domain is $[155 \mathrm{~Hz}, 165 \mathrm{~Hz}$ ], and the searching ranges of the tunable parameter are $k \in[0.1,15], a \in[0.1,10], b \in[0.1,10]$, $\varepsilon \in[5,15]$ and $R \in[5000,20000]$, respectively. It should be pointed out that the search ranges of $\varepsilon$ and $R$ are determined by guaranteeing the amplitude and frequency of the fault signal component after transformation among the appropriate small-parameter range. The waveform and spectrum of the optimal output signal in 10 random optimizations are presented in Figures 10(c) and 10(d), where the optimal parameters are $k=9.3271, a=0.1112, b=0.5065$, $\varepsilon=11.4685$ and $R=6478$, respectively. It can be seen from Figure 10(d) that the signal component of frequency $f_{\mathrm{BPFI}}=158.4 \mathrm{~Hz}$ is evident in the spectrum of output signal due to the greatly enhanced $\mathrm{SNR}_{\text {out }}$ of $-12.78 \mathrm{~dB}$. Moreover, the $3 \mathrm{X}$ and $4 \mathrm{X}$ higher-order harmonics can be observed as well. The output result indicates that an inner ring fault may exist in the drive end rolling bearing, which is accordant with the real physical truth of the rolling bearing. Thus, the adaptive diagnosis of an inner ring fault is realized by using the AMPASR method of Duffing system. Moreover, the same date has been analyzed in [27] using a manual adjusted tristable SR system. The output SNR in that reference $(-25.43 \mathrm{~dB})$ is much smaller than that in Figure 10(d) $(-12.78 \mathrm{~dB})$, thus proving the advantage of the proposed AMPASR method in adaptive rolling bearing inner ring fault diagnosis.

Moreover, the same vibration signal with inner ring fault can be also detected by the proposed AMPASR method of Langevin system. The optimal $\mathrm{SNR}_{\text {out }}$ of the output signals from both Duffing system and Langevin system in 10 random adaptive detections are presented in Figure 11. It can be seen that although the Langevin system is helpful to enhance the $\mathrm{SNR}_{\text {out }}$ of the detected signal, the optimal $\mathrm{SNR}_{\text {out }}$ of the output signals from Langevin system is generally smaller than those from Duffing system. Again, the Duffing system is demonstrated as a better bistable system in 


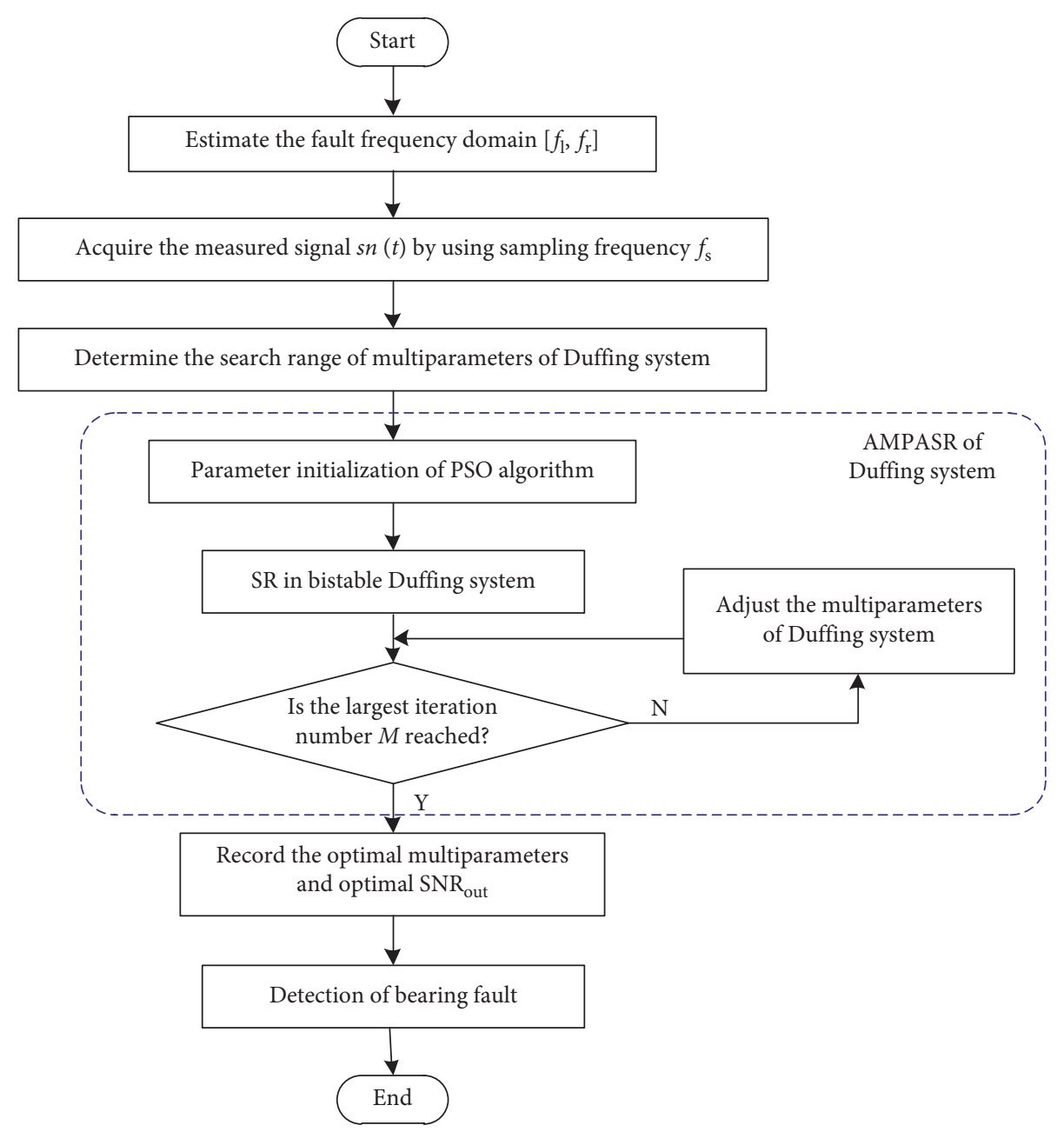

Figure 8: The flowchart of the bearing fault diagnosis based on the AMPASR of Duffing system.

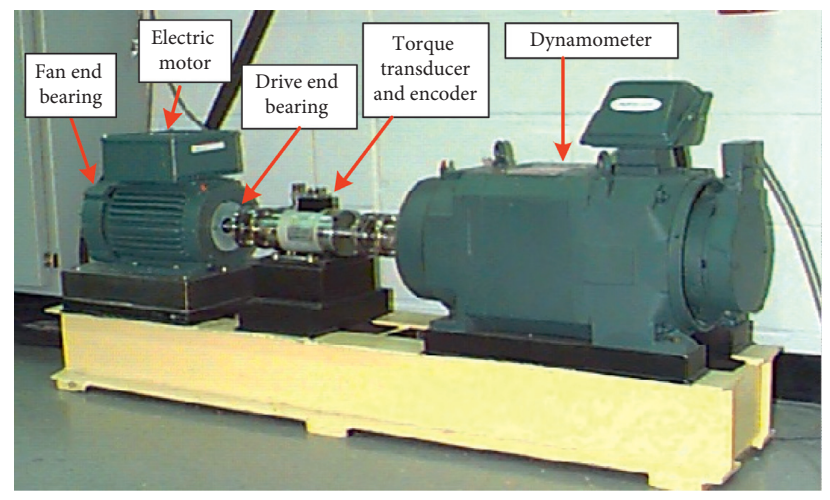

FIGURE 9: CWRU bearing test rig.

TABLE 5: Drive end bearing details and fault frequencies.

\begin{tabular}{|c|c|c|c|c|c|c|c|c|c|}
\hline \multirow[b]{2}{*}{$\begin{array}{l}\text { Position } \\
\text { on rig }\end{array}$} & \multirow[b]{2}{*}{$\begin{array}{l}\text { Model } \\
\text { number }\end{array}$} & \multirow{2}{*}{$\begin{array}{c}\text { Inside } \\
\text { diameter } \\
\text { (inches) }\end{array}$} & \multirow{2}{*}{$\begin{array}{l}\text { Outside } \\
\text { diameter } \\
\text { (inches) }\end{array}$} & \multirow[b]{2}{*}{$\begin{array}{l}\text { Thickness } \\
\text { (inches) }\end{array}$} & \multirow[b]{2}{*}{$\begin{array}{l}\text { Ball diameter } \\
\text { (inches) }\end{array}$} & \multirow{2}{*}{$\begin{array}{l}\text { No. of } \\
\text { rolling } \\
\text { elements }\end{array}$} & \multicolumn{3}{|c|}{$\begin{array}{c}\text { Fault frequencies } \\
\text { (multiple of shaft speed) }\end{array}$} \\
\hline & & & & & & & $\begin{array}{l}\text { Inner } \\
\text { ring } \\
f_{\text {BPFI }}\end{array}$ & $\begin{array}{c}\text { Outer } \\
\text { ring } \\
f_{\mathrm{BPFO}}\end{array}$ & $\begin{array}{c}\text { Rolling } \\
\text { element } \\
f_{\mathrm{BPFB}}\end{array}$ \\
\hline Drive end & $\begin{array}{c}\text { SKF 6205- } \\
\text { 2RS JEM }\end{array}$ & 0.9843 & 2.0472 & 0.5906 & 0.3126 & 9 & 5.4152 & 3.5848 & 4.7135 \\
\hline
\end{tabular}




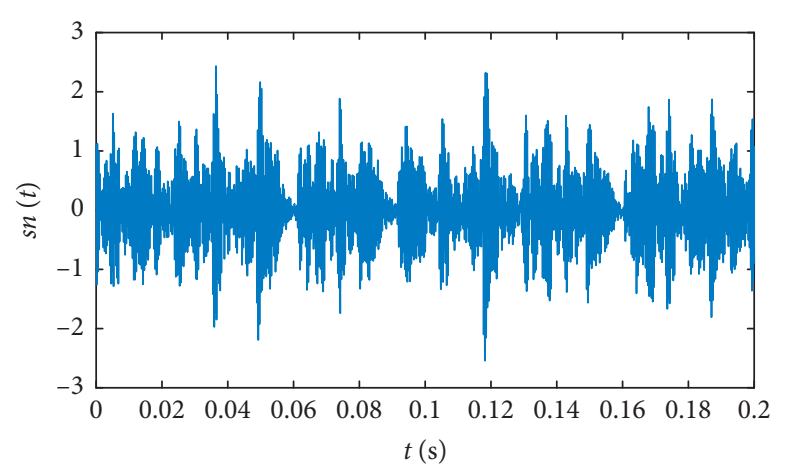

(a)

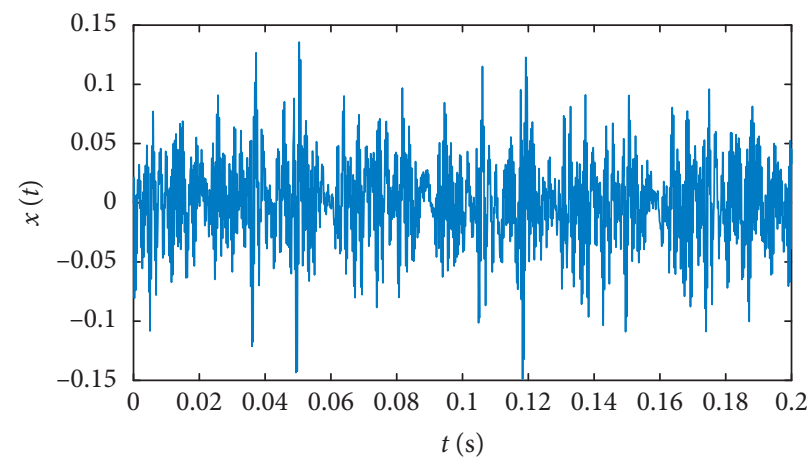

(c)

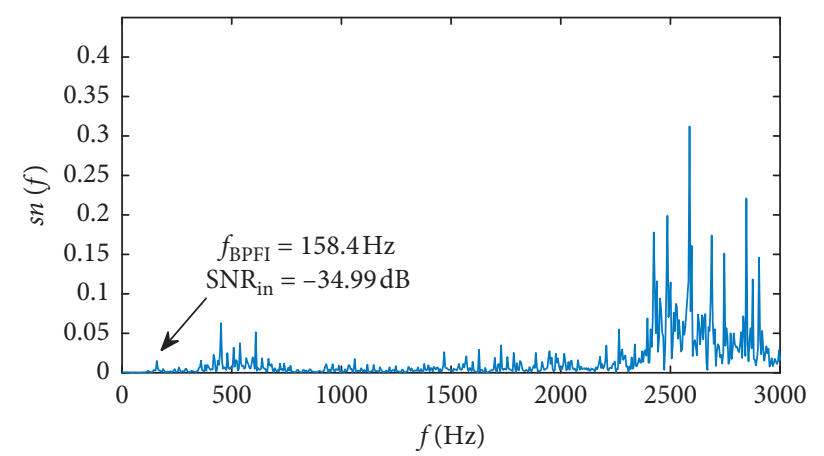

(b)

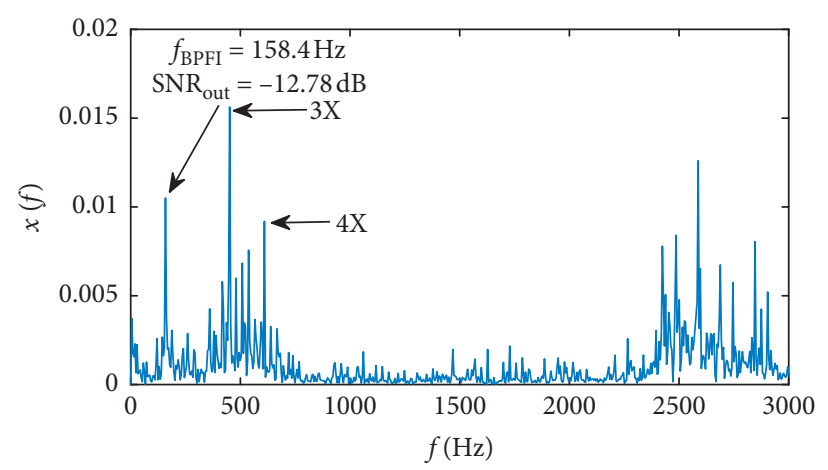

(d)

FIGURE 10: Adaptive detection results of inner ring fault. (a) Waveform and (b) spectrum of the input signal. (c) Waveform and (d) spectrum of the output signal.

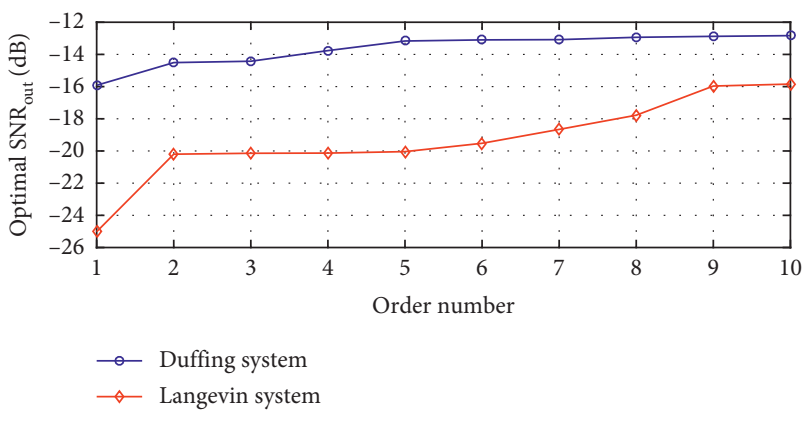

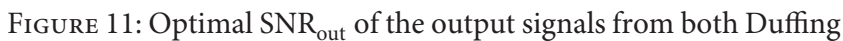
system and Langevin system in 10 random adaptive detections.

weak-signal detection compared with the Langevin system. Thus, in the following fault diagnosis cases, we only study the detection results from the Duffing system.

5.2.2. Case 2: Outer Ring Fault Diagnosis. Similarly, the fault frequency of an outer ring fault can be calculated as $f_{\mathrm{BPFO}} \approx 106.0 \mathrm{~Hz}$ according to Table 5 . The waveform and spectrum of the original vibration signal with outer ring fault, which is 0.021 inches in diameter, are presented in Figures 12 (a) and 12(b). It can be seen that the signal component of outer ring fault frequency $f_{\mathrm{BPFO}} \approx 106.0 \mathrm{~Hz}$ cannot be identified in both the waveform and the spectrum due to the extremely low input SNR $\left(\mathrm{SNR}_{\mathrm{in}}=-53.16 \mathrm{~dB}\right)$.
Again, the proposed AMPASR method of Duffing system is adopted to analyze the vibration signal. In each optimization, the potential frequency domain is $[102 \mathrm{~Hz}, 110 \mathrm{~Hz}]$, and the searching ranges of the tunable parameter are $k \in[0.1,15], a \in[0.1,10], b \in[0.1,10]$, $\varepsilon \in[5,15]$ and $R \in[5000,20000]$, respectively. The waveform and spectrum of the optimal output signal in 10 random optimizations are presented in Figures 12(c) and $12(\mathrm{~d})$, where the optimal parameters are $k=1.1764$, $a=4.9527, b=5.7666, \varepsilon=8.6071$ and $R=10522$, respectively. One can see that the signal component of frequency $f_{\mathrm{BPFO}}=105.6 \mathrm{~Hz}$ can be identified from the spectrum of output signal shown in Figure $12(\mathrm{~d})$, where $\mathrm{SNR}_{\text {out }}$ is enhanced to $-14.64 \mathrm{~dB}$. This result indicates the existence of an outer ring fault in the drive end rolling bearing. Thus, the adaptive diagnosis of an outer ring fault is realized by using the AMPASR method of Duffing system.

5.2.3. Case 3: Rolling Element Fault Diagnosis. At last, the vibration signal with a rolling element fault is considered. The fault frequency of a rolling element fault can be calculated as $f_{\mathrm{BPFB}} \approx 139.4 \mathrm{~Hz}$ according to Table 5 . The waveform and spectrum of original vibration signal with rolling element fault, which is 0.021 inches in diameter, are presented in Figures 13(a) and 13(b), where the signal component of rolling element fault frequency $f_{\mathrm{BPFB}} \approx 139.4 \mathrm{~Hz}$ is completely submerged due to the extremely low input SNR $\left(\mathrm{SNR}_{\text {in }}=-49.23 \mathrm{~dB}\right)$. 


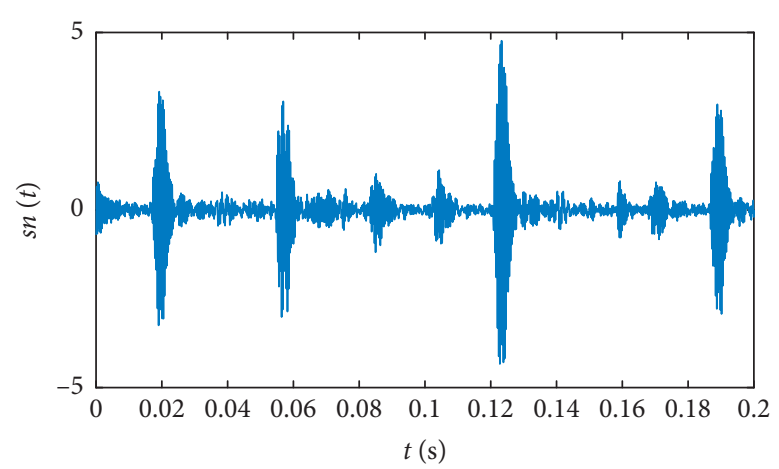

(a)

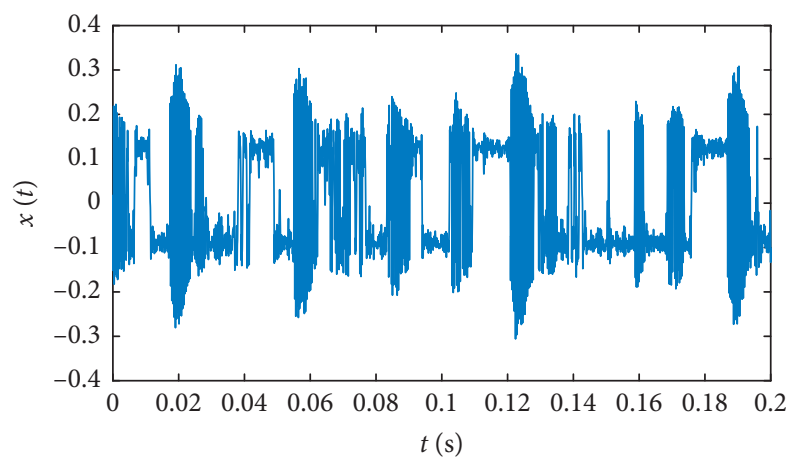

(c)

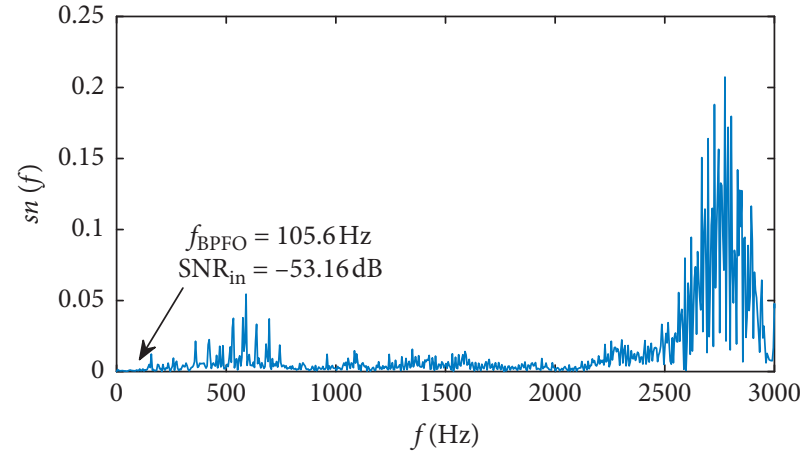

(b)

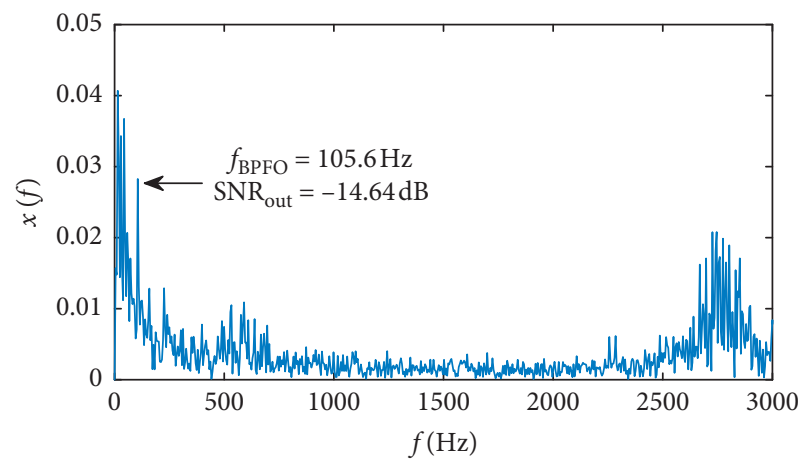

(d)

FIGURE 12: Adaptive detection results of outer ring fault. (a) Waveform and (b) spectrum of the input signal. (c) Waveform and (d) spectrum of the output signal.

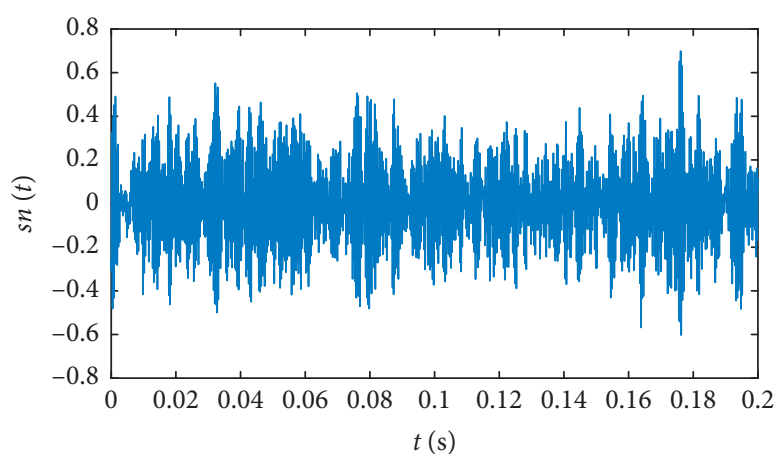

(a)

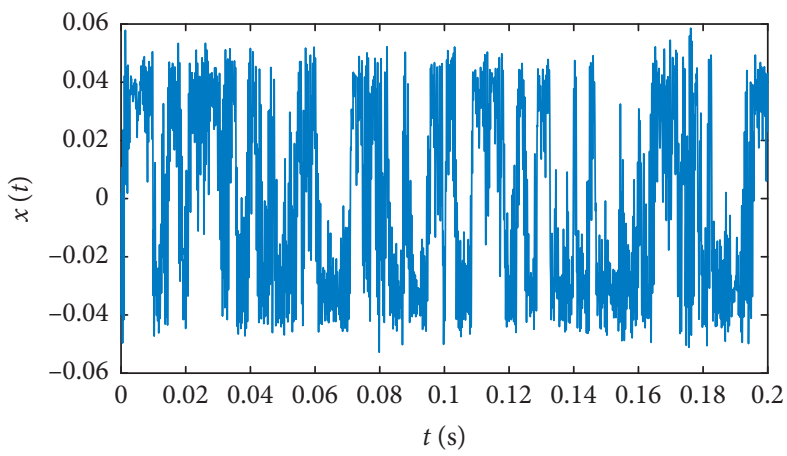

(c)

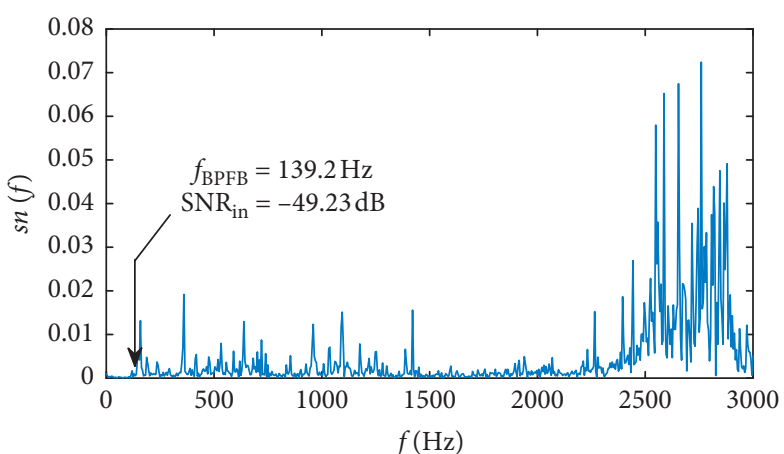

(b)

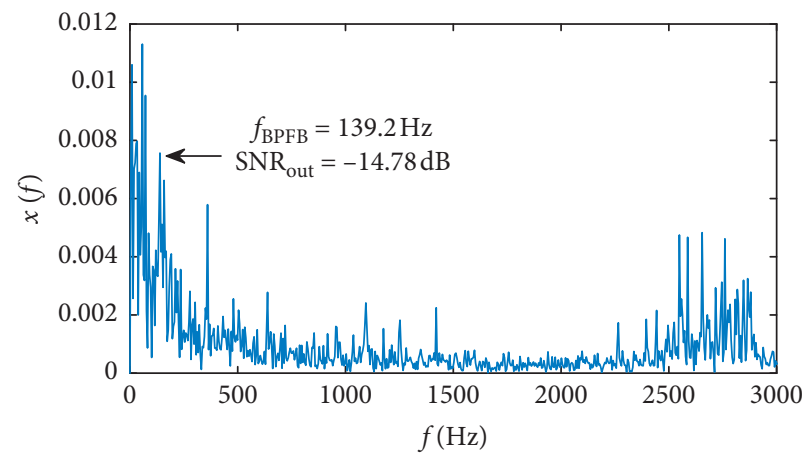

(d)

FIGURE 13: Adaptive detection results of outer ring fault. (a) Waveform and (b) spectrum of the input signal. (c) Waveform and (d) spectrum of the output signal. 
Once again, the proposed AMPASR method of Duffing system is adopted to analyze the vibration signal. In each optimization, the potential frequency domain is $[135 \mathrm{~Hz}, 145 \mathrm{~Hz}]$, and the searching ranges of the tunable parameter are $k \in[0.1,15], a \in[0.1,10], b \in[0.1,10]$, $\varepsilon \in[20,50]$ and $R \in[5000,20000]$, respectively. The waveform and spectrum of the optimal output signal in 10 random optimizations are presented in Figures 13(c) and $13(\mathrm{~d})$, where the optimal parameters are $k=8.0513$, $a=6.1798, b=9.6208, \varepsilon=22.6837$ and $R=12364$, respectively. It can be seen that the signal component of frequency $f_{\mathrm{BPFB}}=139.2 \mathrm{~Hz}$ can be identified from the spectrum of output signal shown in Figure 13(d), where $\mathrm{SNR}_{\text {out }}$ is enhanced to $-14.78 \mathrm{~dB}$. This result indicates that a rolling element fault is in the drive end rolling bearing. Thus, the adaptive diagnosis of a rolling element fault is realized by using the AMPASR method of Duffing system.

5.3. Discussion of Practical Examples. In this section, the feasibility of the proposed weak-signal detection method based on AMPASR of Duffing system has been demonstrated. It can be seen that the weak features of the inner ring, outer ring, and rolling elements faults can be greatly enhanced by adjusting the system parameters adaptively to produce an optimal system output SR. Moreover, the calculation time with the given optimization parameters is around $6 \mathrm{~s}$, which is acceptable in practical engineering. Therefore, the proposed approach provides a reliable solution for both online and offline adaptive diagnosis of rolling bearing faults.

Actually, the length of the detected vibration signal $T$ has an influence on the detection results, especially for the online detections. It is well known that $T=N / f_{s}$. The changes of $T$ indicate two cases: (1) When $N$ is fixed and $f_{s}$ is set according to the characteristic frequency $f_{0}$ of different vibration fault signals, we can simply change the searching range of the scale-transformation coefficient $R$ to get a good optimization result; (2) when $f_{s}$ is fixed for a given vibration fault signal, the change of $N$ indicates the change of $T$. On one hand, when $N(T)$ is smaller, it costs less time for the adaptive optimization process and the detection is more efficient especially for online detection. On the other hand, when $N(T)$ is larger, more information is involved in the signal and the spectrum resolution $f_{s} / N$ is larger, which is important for some complicated signals. Therefore, we should set the value of $N$ appropriately especially for online detections, or we can set different values of $N$ to deal with the same vibration fault signal to find the best output result.

It should be pointed out that we always assumed that the fault types of rolling bearing are already known in the above examples. Thus, the corresponding fault frequencies, which are necessary for adaptive adjustments, can be calculated, and the feasibility of the proposed method can be verified. However, in most cases of practical engineering, the existence of fault and the potential fault type cannot be foreseen. Therefore, one should calculate the fault frequencies of the inner ring, outer ring, and rolling elements based on the rotating frequency of the motor. Potential frequency domains are selected accordingly to obtain the optimal output result for each fault type. Thus, the existence of the three rolling fault types can be recognized and the fault detection can be realized.

In this section, we only consider the cases of single fault to demonstrate the feasibility of the proposed adaptive method. However, the weak vibration signals with a multifault signal are quite common in practical engineering. For these cases, one can select different frequency domains for different fault frequencies to realize the detections of multifaults. This is worthy of further study in the near future.

\section{Conclusion}

Aiming at the fault diagnosis of rolling bearings, an adaptive multiparameter-adjusting SR method for bistable systems based on PSO algorithm has been proposed and studied in this paper. The tunable parameters include the traditional nonlinear system parameters and other generalized parameters such as the amplitude-transformation coefficient and the scale-transformation coefficient. With the output $\mathrm{SNR}\left(\mathrm{SNR}_{\mathrm{out}}\right)$ as objective function, the optimal SR output of bistable systems can be obtained by adjusting the multiparameters adaptively for parameter-fixed vibration signals, and a larger SNR can be achieved in the output signal. Thus, potential weak rolling bearing fault features can be extracted using the proposed method and fault diagnosis can be realized. Simulation results show that although the adaptive method can only find out a local optimal solution in each optimization, the obtained $\mathrm{SNR}_{\text {out }}$ is close to the optimal one and the weak signal feature can be extracted from the SR output. Based on the proposed AMPASR method, the influence of PSO algorithm parameters on the simulation results is studied, and a set of reasonable algorithm parameters including particles' number and largest iteration number is chosen for further simulations. Furthermore, the optimization results in the Langevin system and Duffing system are compared, and results show that the Duffing system, which is selected in this paper for adaptive fault diagnosis, is a superior bistable system in weak-signal detection. At last, a weak-signal detection method based on AMPASR of the Duffing system is proposed. This method is employed to adaptively diagnose the rolling bearing faults including inner ring fault, outer ring fault, and rolling element fault based on the data from CWRU, thus demonstrating the availability and reliability of the proposed method.

\section{Data Availability}

The data analyzed in Section 5 can be accessed from the Case Western Reserve University Bearing Data Center Website (http://csegroups.case.edu/bearingdatacenter/pages/downloaddata-file).

\section{Conflicts of Interest}

The authors declare that there are no conflicts of interest regarding the publication of this paper. 


\section{Acknowledgments}

This work was supported by National Natural Science Foundation of China (Grant no. 51905349), Natural Science Foundation of Jiangxi Province (CN) (Grant no. 20161BAB216111), Natural Science Foundation of Shenzhen University (Grant no. 2019036, and 860-000002110264), and Key Laboratory of Lightweight and High Strength Structural Materials of Jiangxi Province (Grant no. 20171BCD40003).

\section{References}

[1] F. Jia, Y. Lei, H. Shan, and J. Lin, "Early fault diagnosis of bearings using an improved spectral kurtosis by maximum correlated kurtosis deconvolution," Sensors, vol. 15, no. 11, pp. 29363-29377, 2015.

[2] Y. Wang, J. Xiang, R. Markert, and M. Liang, "Spectral kurtosis for fault detection, diagnosis and prognostics of rotating machines: a review with applications," Mechanical Systems and Signal Processing, vol. 66-67, pp. 679-698, 2016.

[3] Z. Feng, D. Zhang, and M. J. Zuo, "Adaptive mode decomposition methods and their applications in signal analysis for machinery fault diagnosis: a review with examples," IEEE Access, vol. 5, pp. 24301-24331, 2017.

[4] J. Chen, Z. Li, J. Pan et al., "Wavelet transform based on inner product in fault diagnosis of rotating machinery: a review," Mechanical Systems and Signal Processing, vol. 70-71, pp. 1-35, 2016.

[5] H. Shi and W. Li, "The application of chaotic oscillator in detecting weak resonant signal of mems resonator," Review of Scientific Instruments, vol. 88, no. 5, Article ID 055003, 2017.

[6] D. Han, X. Su, and P. Shi, "Weak fault signal detection of rotating machinery based on multistable stochastic resonance and VMD-AMD," Shock and Vibration, vol. 2018, Article ID 4252438, 15 pages, 2018.

[7] R. Benzi, A. Sutera, and A. Vulpiani, "The mechanism of stochastic resonance," Journal of Physics A-Mathematical and General, vol. 14, no. 11, pp. 453-457, 1981.

[8] L. Gammaitoni, P. Hänggi, P. Jung, and F. Marchesoni, "Stochastic resonance," Reviews of Modern Physics, vol. 70, no. 1, pp. 223-287, 1998.

[9] C. U. Mba, V. Makis, S. Marchesiello, A. Fasana, and L. Garibaldi, "Condition monitoring and state classification of gearboxes using stochastic resonance and hidden markov models," Measurement, vol. 126, pp. 76-95, 2018.

[10] S. Zhong, L. Zhang, H. Wang, H. Ma, and M. Luo, "Nonlinear effect of time delay on the generalized stochastic resonance in a fractional oscillator with multiplicative polynomial noise," Nonlinear Dynamics, vol. 89, no. 2, pp. 1327-1340, 2017.

[11] Y. Zhang, Y. Jin, and P. Xu, "Stochastic resonance and bifurcations in a harmonically driven tri-stable potential with colored noise," Chaos, vol. 29, no. 2, Article ID 023127, 2019.

[12] J. Yang, M. A. F. Sanjuan, P. Chen, and H. Liu, "Stochastic resonance in overdamped systems with fractional power nonlinearity," European Physical Journal Plus, vol. 132, no. 10, p. 432, 2017.

[13] K. Yang, Z. Zhang, Y. Zhang, and H. Huang, "High-resolution monitoring of aerospace structure using the bifurcation of a bistable nonlinear circuit with tunable potential-well depth," Aerospace Science and Technology, vol. 87, pp. 98-109, 2019.

[14] S. Zhou, J. Cao, D. J. Inman, J. Lin, S. Liu, and Z. Wang, "Broadband tristable energy harvester: modeling and experiment verification," Applied Energy, vol. 133, pp. 33-39, 2014.
[15] Y. Gao, Y. Leng, A. Javey et al., "Theoretical and applied research on bistable dual-piezoelectric-cantilever vibration energy harvesting toward realistic ambience," Smart Materials and Structures, vol. 25, no. 11, p. 115032, 2016.

[16] G. Hu, J. L. Wang, Z. Su, G. P. Peng, and K. C. S. Kwok, "Performance evaluation of twin piezoelectric wind energy harvesters under mutual interference," Applied Physicas Letter, vol. 115, no. 7, Article ID 073901, 2019.

[17] Z. Qiao, Y. Lei, and N. Li, "Applications of stochastic resonance to machinery fault detection: a review and tutorial," Mechanical Systems and Signal Processing, vol. 122, pp. 502536, 2019.

[18] S. Lu, Q. He, and J. Wang, "A review of stochastic resonance in rotating machine fault detection," Mechanical Systems and Signal Processing, vol. 116, pp. 230-260, 2019.

[19] N. V. Agudov, A. V. Krichigin, D. Valenti, and B. Spagnolo, "Stochastic resonance in a trapping overdamped monostable system," Physical Review E, vol. 81, no. 5, Article ID 051123, 2010.

[20] M. Yao, W. Xu, and L. Ning, "Stochastic resonance in a bias monostable system driven by a periodic rectangular signal and uncorrelated noises," Nonlinear Dynamics, vol. 67, no. 1, pp. 329-333, 2012.

[21] Y. Qin, Y. Tao, Y. He, and B. Tang, "Adaptive bistable stochastic resonance and its application in mechanical fault feature extraction," Journal of Sound and Vibration, vol. 333, no. 26, pp. 7386-7400, 2014.

[22] J. Li, J. Zhang, M. Li, and Y. Zhang, "A novel adaptive stochastic resonance method based on coupled bistable systems and its application in rolling bearing fault diagnosis," Mechanical Systems and Signal Processing, vol. 114, pp. 128-145, 2019.

[23] G. Zhang, Y. Zhang, T. Zhang, and J. Xiao, "Stochastic resonance in second-order underdamped system with exponential bistable potential for bearing fault diagnosis," IEEE Access, vol. 6, pp. 42431-42444, 2018.

[24] J. Hao, T. Du, C. Jiang, S. Sun, and C. Fu, “Application of parameter-tuning stochastic resonance for detecting weak signal with ultrahigh frequency," Journal of Computer Applications, vol. 36, no. 9, pp. 2374-2380, 2016.

[25] Z. Lai and Y. Leng, "Weak-signal detection based on the stochastic resonance of bistable duffing oscillator and its application in incipient fault diagnosis," Mechanical Systems and Signal Processing, vol. 81, pp. 60-74, 2016.

[26] Z. Lai and Y. Leng, "Generalized parameter-adjusted stochastic resonance of duffing oscillator and its application to weak-signal detection," Sensors, vol. 15, no. 9, pp. 2132721349, 2015.

[27] Z. Lai, J. Liu, H. Zhang, C. Zhang, J. Zhang, and D. Duan, "Multi-parameter-adjusting stochastic resonance in a standard tri-stable system and its application in incipient fault diagnosis," Nonlinear Dynamics, vol. 96, no. 3, pp. 2069-2085, 2019.

[28] L. Tong, X. Li, J. Hu, and L. Ren, “A PSO optimization scaletransformation stochastic-resonance algorithm with stability mutation operator,” IEEE Access, vol. 6, pp. 1167-1176, 2018.

[29] P. Xia, H. Xu, M. Lei, and Z. Ma, "An improved stochastic resonance method with arbitrary stable-state matching in underdamped nonlinear systems with a periodic potential for incipient bearing fault diagnosis," Measurement Science and Technology, vol. 29, no. 8, Article ID 085002, 2018.

[30] J. Lu, M. Huang, and J.-J. Yang, "A novel spectrum sensing method based on tri-stable stochastic resonance and quantum particle swarm optimization," Wireless Personal Communications, vol. 95, no. 3, pp. 2635-2647, 2017. 
[31] P. Zhou, S. Lu, F. Liu, Y. Liu, G. Li, and J. Zhao, "Novel synthetic index-based adaptive stochastic resonance method and its application in bearing fault diagnosis," Journal of Sound and Vibration, vol. 391, pp. 194-210, 2017.

[32] S. Wang, F. Wang, S. Wang, and G. Li, "Detection of multifrequency weak signals with adaptive stochastic resonance system," Chinese Journal of Physics, vol. 56, no. 3, pp. 9941000, 2018.

[33] C. He, H. Li, Z. Li, and X. Zhao, "An improved bistable stochastic resonance and its application on weak fault characteristic identification of centrifugal compressor blades," Journal of Sound and Vibration, vol. 442, pp. 677-697, 2019.

[34] X. Zhang, J. Wang, Z. Liu, and J. Wang, "Weak feature enhancement in machinery fault diagnosis using empirical wavelet transform and an improved adaptive bistable stochastic resonance," Isa Transactions, vol. 84, pp. 283-295, 2019.

[35] J. Li, X. Chen, and Z. He, "Adaptive stochastic resonance method for impact signal detection based on sliding window," Mechanical Systems and Signal Processing, vol. 36, no. 2, pp. 240-255, 2013.

[36] Y. Wang, S. Jiao, Q. Zhang, S. Lei, and X. Qiao, "A weak signal detection method based on adaptive parameter-induced tristable stochastic resonance," Chinese Journal of Physics, vol. 56, no. 3, pp. 1187-1198, 2018.

[37] J. Liu, Y. Leng, Z. Lai, and S. Fan, "Multi-frequency signal detection based on frequency exchange and re-scaling stochastic resonance and its application to weak fault diagnosis," Sensors, vol. 18, no. 5, p. 1325, 2018.

[38] W. A. Smith and R. B. Randall, "Rolling element bearing diagnostics using the case western reserve university data: a benchmark study," Mechanical Systems and Signal Processing, vol. 64-65, pp. 100-131, 2015. 